\title{
Commercial Acetic Acid: A Potential Regenerative Agent for Chronic Cutaneous Wounds? A Quasi-Experimental Study
}

\author{
Ma'an Al-Khalisy ${ }^{1} \&$ Ahmed Al-Imam ${ }^{1,2}$ \\ ${ }^{1}$ Department of Anatomy and Cellular Biology, College of Medicine, University of Baghdad, Iraq \\ ${ }^{2}$ Department of Postgraduate Medicine, School of Life and Medical Sciences, University of Hertfordshire, United \\ Kingdom \\ Correspondence: Dr Ahmed Al-Imam, House 18/5, Al-Akhtal Street, District 318, Al-Adhamyia, 10053, Baghdad, \\ Iraq. E-mail: tesla1452@gmail.com; a.m.al-imam@herts.ac.uk
}

Received: July 25, 2017 Accepted: August 17, 2017 Online Published: September 18, 2017

doi:10.5539/gjhs.v9n11p178 URL: https://doi.org/10.5539/gjhs.v9n11p178

\begin{abstract}
Background: The acetic acid in the form of vinegar has been used for medicinal purposes since ancient times. Acetic acid is reported to be used for medicinal uses at $1-5 \%$ concentration. The mechanism behind the regenerative potential of commercial acetic acid has not been fully explored.

Materials and Methods: Twelve Iraqi patients with chronic skin ulceration were admitted and managed at a Dermatology clinic. Patients included men (7) and women (5) of diverse age groups (23-81 years). The aetiology behind the skin ulceration included; diabetic ulcer, decubitus ulcer, and others induced by trauma, bone fracture, insect bites and substance abuse. All patients were strictly treated with commercial acetic acid (at 4-6\% concertation) and followed-up in time until the complete resolution of their cutaneous ulcerations.
\end{abstract}

Results and Discussion: This study is quasi-experimental; two cases are discussed thoroughly; the first case belongs to a Caucasian male aged 26 years with schizophrenia and addiction towards psychoactive substances; he developed a refractory ulcer in left cubital fossa following an intravenous injection. The second case belongs to a middle age male patient, hypertensive otherwise healthy; he had a displaced intra-articular fracture affecting the distal end of the left radius; it was surgically treated with an internal fixation which was incriminated for the development of an ulcer over the volar aspect of left forearm overlying the internal fixation site. Local disinfection with commercial apple vinegar was very effective in restoring the skin integrity with minimal scarring.

Conclusion: The regenerative potential of acetic acid has been marginally covered in the literature. The presented cases indicate a regenerative potential or at least an accelerant role in the process of cutaneous wound healing and repair.

Keywords: Acetic acid, vinegar, wound healing, ulcer, burns, disinfectant, antiseptic, antibacterial, antimicrobial, angiogenic, mitogens, regeneration, re-epithelialization

\section{Background}

Acetic acid (AA) has been used as an antiseptic and antimicrobial agent dating back to the $2^{\text {nd }}$ millennium BC. It has been utilised for the treatment of cutaneous wounds, superficial and deep ulcer, including decubitus and neuropathic ulcers (Fraise et al., 2013; Phillips et al., 1968). Acetic acid, also known as anhydrous acetic acid, is a weak organic acid with the chemical formula $\mathrm{CH} 3 \mathrm{COOH}$ (Figure 1) (Molview.org, 2016). It is a colourless liquid that when undiluted, becomes known as glacial acetic acid (GAA). Acetic acid is a weak acid. However, it is highly dangerous to skin when concentrated, and it becomes corrosive at a concentration above $25 \%$, and corrosive-flammable at a level above 90\% (SIGMA-ALDRICH, A Part of Merck, 2016; TOXBASE, 2016). 


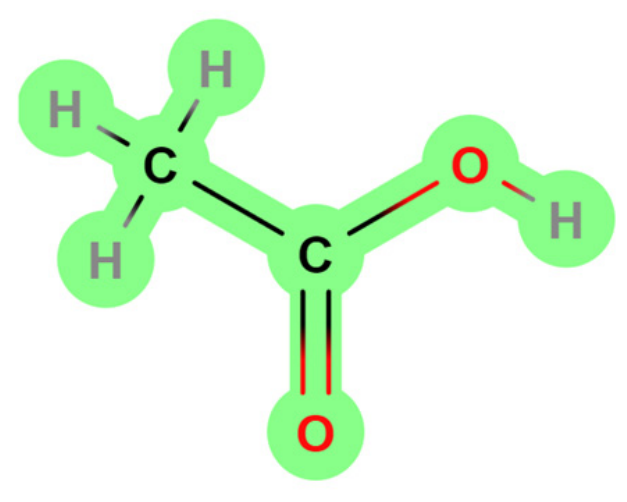

Figure 1. The Molecular Structure of Acetic Acid

It is well documented that thieves had used commercial acetic acid (vinegar) during the great plague of Europe, in order to avoid contracting an infection while vandalising and stealing valuables from the dead corpse of the plague victims (Fraise et al., 2013). Similarly, during the American Civil War and the $1^{\text {st }}$ World War, the apple cider vinegar was implemented in wound dressings. Later on, acetic acid has been a widely used as a topical antiseptic for the treatment of burns, skin wounds and ulcers. Besides, it has been very well known to have an excellent antimicrobial activity against Gram-negative organisms, particularly for the stubborn Pseudomonas aeruginosa, and resistant strains of both Pseudomonas aeruginosa and Staphylococcus aureus (Fraise et al., 2013; Phillips et al., 1968). However, the popularity of acetic acid in wound dressing has waned considerably in the past decades, due to the development of advanced and experimental dressings, and more recently biological and composite dressings, synthetic skin grafts, and dermal substitutes (Fraise et al., 2013; Valle et al, 2014; Walter et al., 2012).

Unlike other antiseptic agents and despite the volatility of acetic acid, Fraise and colleagues have found that $5 \%$ acetic acid to be very stable chemically when applied directly to wounds or as part of the dressing. The acid's evaporation during the first 24 hours after application, has a negligible impact on its disinfectant properties. Furthermore, these properties were not inhibited by organic material such as cotton. Another advantage over other disinfectants-antimicrobials is that vinegar is inexpensive and easy-to-purchase, which is of prime value in economically-backwards and developing countries (Fraise et al., 2013; Valle et al., 2014; Walter et al., 2012). Acetic acid had also been used as a solvent and a co-agent of purification in a vast plethora of chemical reactions (Pickart, 1991).

Non-industrial (commercial) acetic acid was used and provided results that may indicate a potential regenerative ability in treating chronic infected wounds and ulcers. The level-of-evidence of this manuscript is level-3 in accordance with the Oxford Centre for Evidence-based Medicine (Oxford Centre for Evidence-based Medicine, 2009). The literature review was done systematically across PubMed/Medline, the Cochrane Library, Scopus, Embase, and Google Scholar. The search strategy was based on an elaborate combination of keyword applied across medical and paramedical databases. The keyword used were: "Glacial Acetic acid", "Acetic acid", "Topical Agent", "Chronic wounds", "Ulcer", "Burn", "Antiseptic", “Antibacterial”, “Antimicrobial”, "Angiogenic Activity", "Mitogen", "Regeneration", "Skin", "Cytotoxicity", and "Pseudomonas aeruginosa". A combination of these keywords was also applied using Boolean Operators. (Lee et al., 1993). There was a paucity of literature on the regenerative and mitogenic potentials of this anciently-used chemical substance (Banda et al., 1982).

\section{Materials and Methods}

The study is quasi-experimental in connection to a case series of patients with chronic cutaneous ulceration. Twelve patients, aged 23 to 81 years, have been managed medically managed via the topical application of commercial acetic acid refined from apple at a concentration of 4-6\%. The patients, seven men and five women, were followed up in time for a variable period of between two weeks and up to two months. The aetiology behind the chronic cutaneous ulcerations in those patients was diverse including; decubitus ulcer $(\mathrm{n}=2)$, venous ulcer (5), traumatic ulcer (1), diabetic ulcer (1), bone fracture-related ulcer (1), Insect bite-related ulcer (1), and IV-injection related ulcer (1). Further, multiple pathologies coexisted in those patients including; substance abuse and addiction, chronic hypertensive disorder, congestive heart failure (CHF), type-1 and type-2 diabetes mellitus (DM), and bone fractures (femoral and radial fractures). Therefore, these cases are considered to be complicated and challenging to be managed. Additionally, commercial Acetic acid was strictly used to manage those refractory ulcers. 
The commercial Acetic acid, refined from Apple at 4-6\% concentration, was applied to each ulcer three times a day after cleansing the ulcer with distilled water. The evolution of the ulcerative wound was followed up at regular intervals either once or twice weekly depending on the medical case. The follow-up period was to monitor the ulcer evolution including; the formation of a healthy granulation tissue, re-epithelialization and wound closure. Patients were also examined regularly for their vital signs, blood glucose levels, in addition to other collateral investigations tailored specifically for each individual case. Moreover, wound bed has been swabbed once weekly for the purpose of assessing the status of bacterial colonization and contamination.

The total number of reference materials cited in this literature are sixteen; the level-of-evidence of this manuscript is estimated to be of level-3 in accordance with the categorization scheme imposed by the Oxford Centre for Evidence-based Medicine (CEBM) (Oxford Centre for Evidence-based Medicine, 2009). The literature review was done systematically across PubMed/Medline, Embase, CINAHL, the Cochrane Library, Scopus, and Google Scholar. The Grey (unpublished) literature was also consulted for relevant data.

\section{Results}

\subsection{CASE-1}

A twenty-six years old Caucasian male from Germany; he is completely healthy apart from his addiction Behaviour (substance use and misuse) towards a subset of illicit drugs known as novel psychoactive substances (NPS) (Al-Imam et al., 2016; Al-Imam, 2017; Al-Imam et al., 2017). He started using these substances as early as the age of 16 years; he used a variety of psychostimulants including LSD, methamphetamines, NBOMe, and DMT. At the age of 22, the patient started developing features of depression, social withdrawal, and paranoid behaviour. At the age of 23 years, he started experiencing hallucinations, mainly auditory and to a less extent visual hallucinations. Further, his paranoid delusions became more consolidated.

The patient was later diagnosed with schizophrenia for which he received the proper psychiatric management including treatment with a medicinal phenothiazine, olanzapine, at a dose between 10-30 mg adjusted in accordance with the severity of his schizophrenic manifestations. Within few months from this management, he started improving and his schizophrenia, including his paranoid behaviour and hallucinations, was in remission. However, the patient was still self-medicating (abusing) NPS, specifically with LSD and NBOMe compounds, which are known to be potent hallucinogenic chemicals (psychedelics and entheogens). Hence, he used to experience hallucinations during his psychedelic tripping, mainly visual illusions and hallucinations (which is less common in classical schizophrenia), for which he used a 10-15 $\mathrm{mg}$ of olanzapine to dampen the effect of these hallucinations at the end of his trip.

The patient used to administer NPS using nasal sniffers, blotters, tablets and capsules, and injections; he used to have intravenous (IV) injections in his left cubital fossa. At the age of 26 and after some repeated IV injections, he developed an ulcer in the left cubital fossa. For which, he sought help by a dermatologist. The ulcer had characteristics of a venous ulcer with a sloping edge, positioned in relation to the cubital fossa and the basilic vein of the left upper limb; there were no features of arterial compromise in connection with the left upper limb. The ulcer was about 15 millimetres in diameter. The ulcer developed features of infection; bacteriological examinations confirmed the colonisation with Staphylococcus. The ulcer was stubborn and persisted for two months; it resisted the traditional modalities of treatment with debridement, anti-staphylococcal antimicrobials, and wound dressing. The dermatologist soon decided to manage the ulcer strictly with commercial Acetic acid which is refined from Apple; the acid was applied to the ulcer three times a day after cleansing the ulcer with distilled water. Within three weeks, there were signs of improvement including; clean ulcer bed, the formation of a healthy granulation tissue, and normal epithelialization at the edges of the ulcer. The ulcer healed successfully with no scarring within two months from starting this management protocol.

\subsection{CASE-2}

The patient is an Iraqi male aged forty-two years; he presented with an evolving ulcer over the volar aspect of the forearm near the distal end of the radius of the left forearm. The patient is healthy apart from well-controlled hypertension; the hypertensive episodes which can be traced back to six years earlier. Additionally, the patient had no significant events of relevance to his past medical history, past surgical history, and drug (medicinal) history. The patient had a road traffic accident (RTA), in which he had a displaced intra-articular fracture of the left radius, also affecting the styloid process of the radius. The condition was managed surgically via open reduction internal fixation (ORIF) with T-plate and screws (Figure 2). 

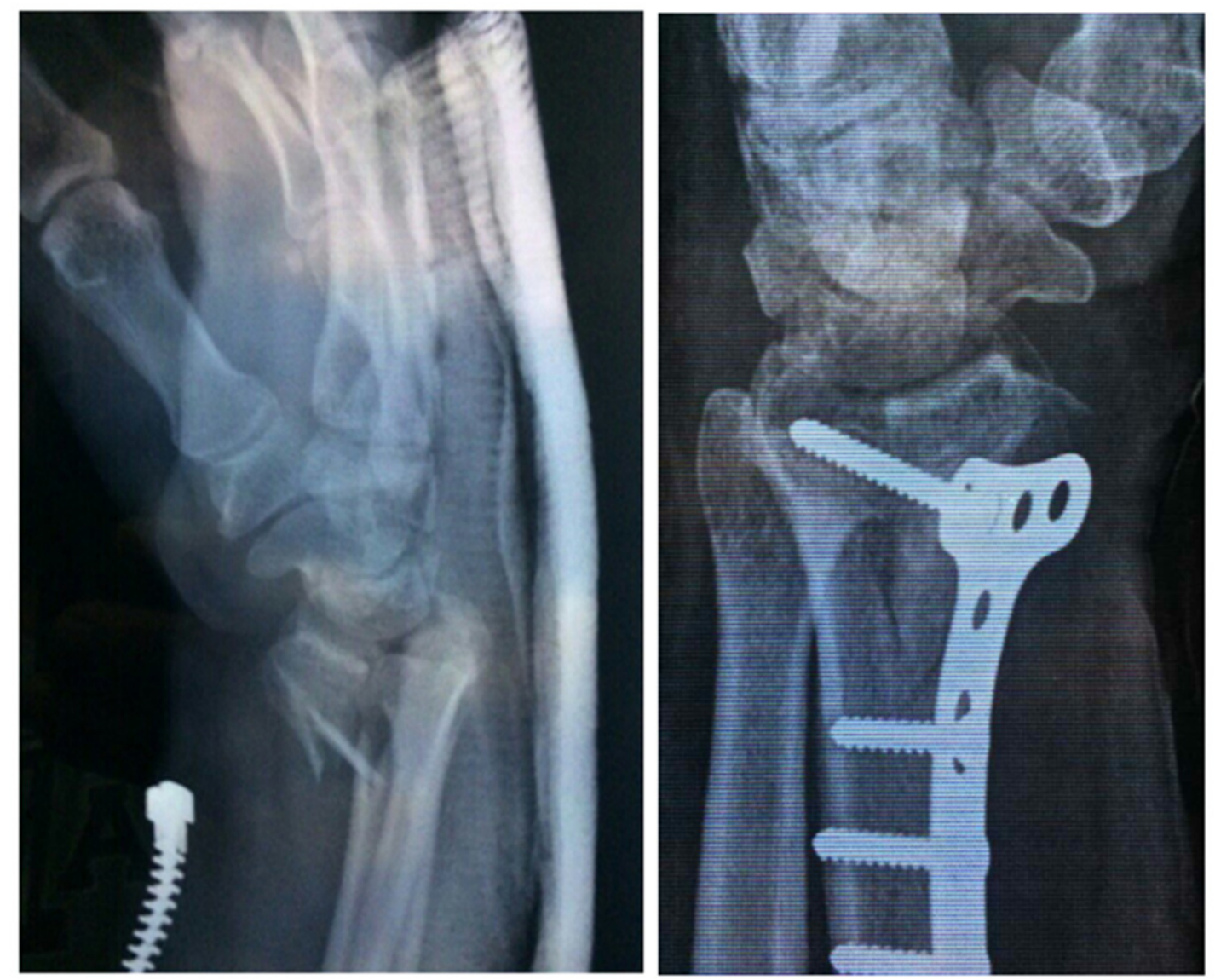

Figure 2. Fracture Site at Distal Radius (left radiograph), Internal Fixation with T-plate and Screws (right)

$\Omega$ The Radiographs have been consented by the patient.

The orthopaedic surgery was successful, although, within few days, the patient started developing skin discolouration followed by erosion and a subsequent ulcer evolution (Figure 3) on the volar aspect of the distal part of the forearm overlying the fixation site. Accordingly, the orthopaedic surgeon decided to refer the patient for a dermatological consultation; the surgeon was also considering the option of removing the T-plate from the fixation site because the fixation might have triggered the ulcer formation. Dermatology consultation confirmed the presence of a moderately-sized ulcer of punched out edges and an unhealthy granulation tissue; the ulcer dimensions were 10 millimetres by 20 millimetres, the longitudinal axis of the ulcer was parallel with the axis of the forearm. It was decided to manage the ulcer conservatively without interfering with the internal fixation; conservative management included the strict application of commercial Acetic acid in the form of apple vinegar. In Iraq, the commercial apple vinegar is sold at an advertised concentration of 4-6\%; it was applied three times each day. Eventually, the ulcer healed successfully (Figure 3) within two weeks; a minimal scar tissue formation can be observed. 

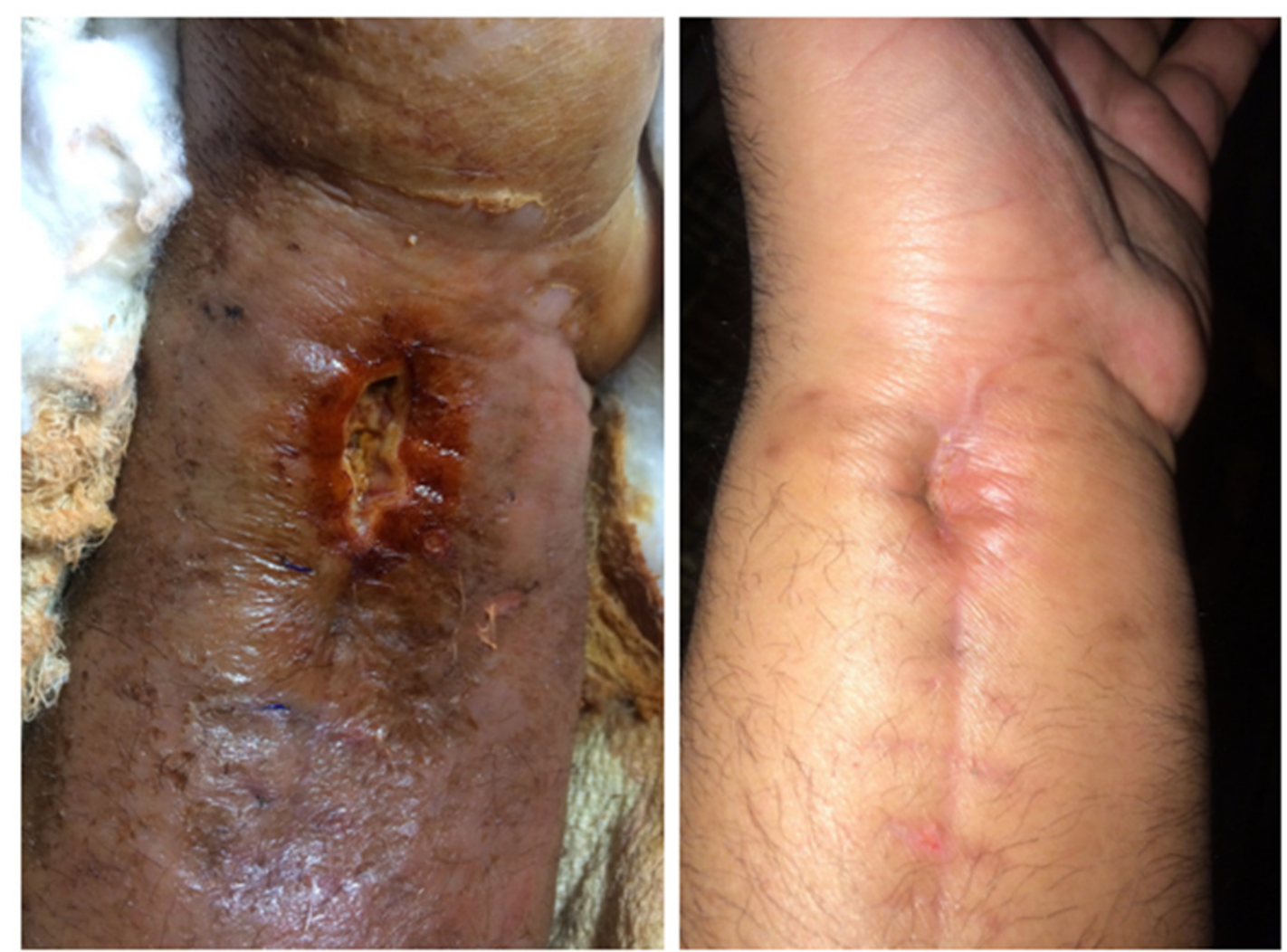

Figure 3. Two Stages of Ulcer's Evolution: Ulcer Formation (left) and Healing (right)

$\Omega$ The photographs have been consented by the patient.

\section{Discussion}

Ryssel and colleagues (2009) using an in-vitro modelling, reported the promptness of $3 \%$ acetic acid; it was used as a local antiseptic agent, particularly for problematic-resistant skin infections caused by Proteus vulgaris, Acinetobacter baumannii, and Pseudomonas aeruginosa (Ryssel et al., 2009). Earlier studies were heterogeneous and based merely on anecdotal data (low level-of-evidence). However, most studies confirmed the effectiveness and safety of the acid at a concentration between $1 \%$ and $5 \%$. Concentrations over $5 \%$ were frequently reported with localised stinginess and unpleasant odour of the substance. Moreover, no significant failures of skin grafts or discomfort were reported in any of those treated patients. However, there were complaints of odour and stinging sensation when the acid was used in concentrations higher than 5\%. Excellent bactericidal effect of acetic acid was verified, particularly against the problematic Gram-negative bacteria such as Pseudomonas aeruginosa and Acinetobacter baumannii. In Proteus Vulgaris, acetic acid was even superior to commercially available antiseptics (Ryssel et al., 2009). Similarly, Nagoba and coworkers (1997), published data on the efficient use of 3\% acetic acid as a strict (solitary) modality of antimicrobial therapy, in which it was applied once daily for 10 days, and the results were very satisfactory (Nagoba et al. 1997).

Additionally, when compared to other antiseptics, it is critical to mention that acetic acid was associated with less frequent side effects and complaints. Moreover, different dilutions of the used disinfectants have a differential effect on the reported claims, while acetic acid can be safely used at a low concentration in the range of $1 \%$ to $5 \%$ (Hirsch et al., 2010; Nagoba et al. 1997). In other words, due to the cytotoxic effect of some antiseptics including acetic acid on human skin cells, it is advised that health care professionals consider the integration of three factors when selecting a disinfectant; the cytotoxicity of the chemical substance (1), the antiseptic properties (2), and the extent (severity) of colonisation of the wound (3) (Hirsch et al., 2010). Very recently, Madhusudhan and coworkers (2015) carried out a randomized controlled trial of thirty-two patients; a lower concentration of Acetic acid (1\%) was proven to be simple, safe, and efficient topical antiseptic for the elimination of Pseudomonas aeruginosa and multi-drug resistant (MDR) Pseudomonas aeruginosa from chronic infected wounds, which was far cheaper than expensive antibiotics, in addition to the fact that it induced no bacterial resistance, and it could be utilized on outpatient basis (Madhusudhan, 2015). 
Nearly four decades ago, Banda and colleagues (1982) reported the isolation of a new angiogenic factor, which was extracted from a rabbit's wound fluid; the authors further stated an interesting fact that signifies the contribution of acetic acid as a co-agent in angiogenic and mitogenic activity, thus promoting efficient and fast wound healing with minimal scarring (Banda et al., 1982). Banda and his colleagues also reported that the acidification ( $\mathrm{PH}$ of 2.9) of the wound fluid by the addition of glacial acetic acid, has enhanced angiogenic activity by 2-6 folds (Banda et al., 1982). Based on systematic review of the literature, this is the first quasi-experimental study from Iraq on the use of commercial acetic acid (vinegar) as an agent of antiseptic, antimicrobial, and potential regenerative properties. Ryssel and colleagues (2009) published a manuscript on the antimicrobial effect of acetic acid; they have stressed on the necessity to study the commercial acetic acid; quoted "It is yet to be proven whether natural Acetic acid has significant benefits with regards to wound healing and bacterial growth". The chemical and molecular mechanism of acetic acid regenerative potential is currently thoroughly investigated via chemical analytics and molecular biology techniques.

This study presents twelve cases of patients with chronic cutaneous ulcerations; patients were of diverse age groups from the $3^{\text {rd }}$ to the $9^{\text {th }}$ decade of life, including both males and females (Table 1). The most common type of ulcer was venous ulceration, and those were found mainly in advanced age groups. The time required for healing following the use of commercial acetic acid was in between two weeks up to two months. Other causes of skin ulceration included decubitus ulcer, diabetic ulcer, traumatic ulceration, and insect bite-related ulcers. Most patients with venous ulcers had other comorbidities including diabetes mellitus (DM), and chronic hypertension. The healing of ulcers and traumatic cutaneous wounds were satisfactory with minimal scar tissue formation. The $1^{\text {st }}$ two cases, as seen in the table, were formerly discussed with details for the purpose of demonstration.

Table 1. Twelve Case of Different Pathologic Ulcerations Managed with Commercial Acetic Acid.

\begin{tabular}{lllll}
\hline Age & Gender & Condition & Time for Healing & Co-existing Conditions \\
\hline 26 & M & IV-injection related ulcer & 2 months & NPS Abuse \\
42 & M & Fracture and ORIF-related ulcer & 2 weeks & Chronic Hypertension \\
26 & F & Decubitus ulcer & 4 weeks & Congestive Heart Failure (CHF) \\
31 & F & Traumatic ulcer & 1.5 month & None \\
67 & M & Venous ulcer & 3 weeks & CHF and Chronic Hypertension \\
81 & F & Venous ulcer & 5 weeks & CHF \\
57 & M & Venous ulcer & 1 month & CHF and type-2 DM \\
23 & M & Insect bite-related ulcer & 2 weeks & None \\
51 & M & Venous ulcer & 4 weeks & CHF \\
23 & F & Decubitus ulcer & 1 month & Fracture of Femur, Bed-ridden \\
26 & F & Diabetic ulcer & 1 month & Type-1 DM \\
71 & M & Venous ulcer & 3 weeks & CHF, DM, and Chronic Hypertension \\
\hline
\end{tabular}

\section{Conclusions}

To be concluded, apple vinegar showed good results by; accelerating wound healing (1), reducing wound contamination and infection (2), exhibiting excellent aesthetic results for the healed wound either with minimal scarring or no scarring at all (3), and having high therapeutic potency to heal wounds contaminated with Pseudomonas aeruginosa, and multi-drug resistant Pseudomonas aeruginosa and Staphylococcus aureus (4). The resultant scar of the healed ulcer revealed an excellent quality comparable to that of a delicate surgical scar. Further, there was no emergence of resistant strains of microbes, unlike the widely used topical and systemic antibiotic, which may induce antibiotic resistance and a subsequent plethora of wound re-infections.

This study proposes a hypothesis concerning the regenerative potential of commercial acetic acid, which has been experimentally suggested by Banda and colleagues (1982). Apple vinegar is cheap, easily-accessible, and possesses a broad spectrum activity against microbes and bacteria. Additionally, it can be easily purchased in economically backwards countries and the developing regions of the world. Moreover, it has few side effects, if any, even at a relatively high concentration. In contrary to the traditionally utilized topical antiseptics. 


\section{Competing Interests Statement}

The authors have nothing to be declared.

\section{Source of Funding}

This study was entirely self-funded.

\section{References}

Al-Imam, A. (2017). Could Hallucinogens Induce Permanent Pupillary Changes in (Ab) users? A Case Report from New Zealand. Case Reports in Neurological Medicine, 2017. https://doi.org/10.1155/2017/2503762

AL-Imam, A., Santacroce, R., Roman - Urrestarazu, A., Chilcott, R., Bersani, G., Martinotti, G., \& Corazza, O. (2017). Captagon: use and trade in the Middle East. Human Psychopharmacology: Clinical and Experimental, $32(3)$.

Al-Imam, A., Simonato, A. P., \& Corazza, O. (2016). Haloperidol, an old antipsychotic with potential use by NPS users in Iraq. Research and Advances in Psychiatry, 3(3), 81-84. Retrieved from https://www.rapjournal.eu/materiale_cic/948_3_3/8031_haloperidol/article.htm

Banda, M. J., Knighton, D. R., Hunt, T. K., \& Werb, Z. (1982). Isolation of a nonmitogenic angiogenesis factor from wound fluid. Proceedings of the National Academy of Sciences, 79(24), 7773-7777. https://doi.org/10.1073/pnas.79.24.7773

Fraise, A. P., Wilkinson, M. A. C., Bradley, C. R., Oppenheim, B., \& Moiemen, N. (2013). The antibacterial activity and stability of acetic acid. Journal of Hospital Infection, 84(4), 329-331. https://doi.org/10.1016/j.jhin.2013.05.001

Hirsch, T., Koerber, A., Jacobsen, F., Dissemond, J., Steinau, H. U., Gatermann, S., ... \& Steinstraesser, L. (2010). Evaluation of toxic side effects of clinically used skin antiseptics in vitro. Journal of Surgical Research, 164(2), 344-350. https://doi.org/10.1016/j.jss.2009.04.029

Lee, J. H., Kin, W. Y., Kim, M. H., \& Lee, Y. J. (1993, July). On the evaluation of Boolean operators in the extended Boolean retrieval framework. In Proceedings of the 16th annual international ACM SIGIR conference on Research and development in information retrieval (pp. 291-297). ACM. https://doi.org/10.1145/160688.160741

Madhusudhan, V. L. (2016). Efficacy of $1 \%$ acetic acid in the treatment of chronic wounds infected with Pseudomonas aeruginosa: prospective randomised controlled clinical trial. International wound journal, 13(6), 1129-1136. https://doi.org/10.1111/iwj.12428

Molview.org. Acetic Acid. Retrieved fromhttp://molview.org/?cid=176 (accessed 11 October 2016).

Nagoba, B. S., Deshmukh, S. R., Wadher, B. J., \& Patil, S. B. (1997). Acetic acid treatment of pseudomonal postoperative wound infection. Journal of Hospital Infection, 36(3), 243-244. https://doi.org/10.1016/S0195-6701(97)90201-2

Oxford Centre for Evidence-based Medicine. Levels of Evidence (March 2009). Retrieved 27 September 2016 from http://www.cebm.net/oxford-centre-evidence-based-medicine-levels-evidence-march-2009/

Pal Singh A. Wrist Injuries X-rays and Photographs. 2016. Retrieved 15 March, 2017, from http://boneandspine.com/wrist-injuries-X-rays-and-photographs/ (accessed).

Phillips, I., Lobo, A. Z., Fernandes, R., \& Gundara, N. S. (1968). Acetic acid in the treatment of superficial wounds infected by Pseudomonas aeruginosa. The Lancet, 291(7532), 11-13. https://doi.org/10.1016/S0140-6736(68)90004-4

Pickart, L. R. (1991). U.S. Patent No. 5,023,237. Washington, DC: U.S. Patent and Trademark Office.

Ryssel, H., Kloeters, O., Germann, G., Schäfer, T., Wiedemann, G., \& Oehlbauer, M. (2009). The antimicrobial effect of acetic acid-an alternative to common local antiseptics?. Burns, 35(5), 695-700. https://doi.org/10.1016/j.burns.2008.11.009

SIGMA-ALDRICH, A Part of Merck. Glacial Acetic Acid. Retrieved 11 October, 2016, from http://www.sigmaaldrich.com/catalog/product/usp/1005706?lang=en\&region=IQ\&cm_sp=Insite-_-prodRec Cold_xviews-_-prodRecCold10-1

TOXBASE. Acetic Acid. Retrieved 11 October, 2016, from https://www.toxbase.org/

Valle, M. F., Maruthur, N. M., Wilson, L. M., Malas, M., Qazi, U., Haberl, E., ... \& Lazarus, G. (2014). 
Comparative effectiveness of advanced wound dressings for patients with chronic venous leg ulcers: a systematic review. Wound Repair and Regeneration, 22(2), 193-204. https://doi.org/10.1111/wrr.12151

Walter, C. J., Dumville, J. C., Sharp, C. A., \& Page, T. (2012). Systematic review and meta - analysis of wound dressings in the prevention of surgical - site infections in surgical wounds healing by primary intention. British Journal of Surgery, 99(9), 1185-1194. https://doi.org/10.1002/bjs.8812

\section{Copyrights}

Copyright for this article is retained by the author(s), with first publication rights granted to the journal.

This is an open-access article distributed under the terms and conditions of the Creative Commons Attribution license (http://creativecommons.org/licenses/by/4.0/). 